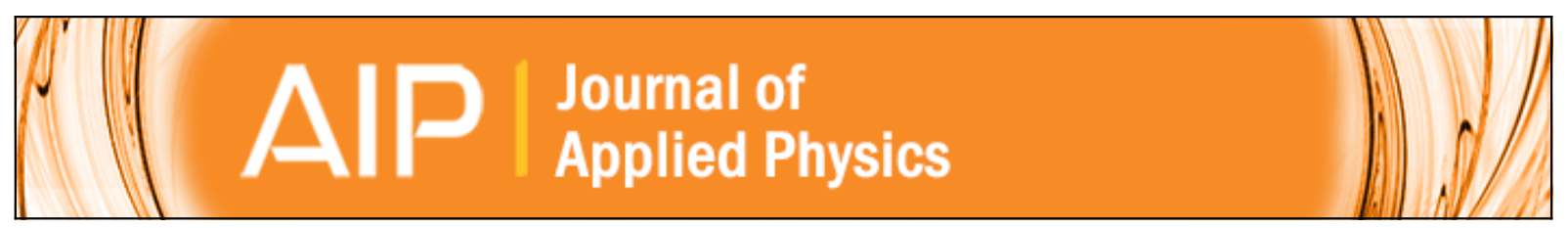

Reduced thermal quenching in indium-rich self-organized InGaN/GaN quantum dots

Rami T. ElAfandy, Tien Khee Ng, Dongkyu Cha, Meng Zhang, Pallab Bhattacharya, and Boon S. Ooi

Citation: Journal of Applied Physics 112, 063506 (2012); doi: 10.1063/1.4751434

View online: http://dx.doi.org/10.1063/1.4751434

View Table of Contents: http://scitation.aip.org/content/aip/journal/jap/112/6?ver=pdfcov

Published by the AIP Publishing

$\underset{\substack{\text { fouburing } \\ \text { R }}}{A}$ Re-register for Table of Content Alerts

Create a profile.

Sign up today! 


\title{
Reduced thermal quenching in indium-rich self-organized InGaN/GaN quantum dots
}

\author{
Rami T. ElAfandy, ${ }^{1}$ Tien Khee Ng, ${ }^{1}$ Dongkyu Cha, ${ }^{2}$ Meng Zhang, ${ }^{3}$ Pallab Bhattacharya,${ }^{3}$ \\ and Boon S. Ooi ${ }^{1, a)}$ \\ ${ }^{1}$ Photonics Laboratory, King Abdullah University of Science and Technology (KAUST), Thuwal 23955-6900, \\ Kingdom of Saudi Arabia \\ ${ }^{2}$ Advanced Nanofabrication, Imaging and Characterization Core Laboratory, King Abdullah University \\ of Science and Technology, Thuwal 23955-6900, Saudi Arabia \\ ${ }^{3}$ Department of Electrical Engineering and Computer Science, University of Michigan, \\ Michigan 48109-2122, USA
}

(Received 28 November 2011; accepted 9 August 2012; published online 18 September 2012)

\begin{abstract}
Differences in optical and structural properties of indium rich (27\%), indium gallium nitride (InGaN) self-organized quantum dots (QDs), with red wavelength emission, and the two dimensional underlying wetting layer (WL) are investigated. Temperature dependent micro-photoluminescence $(\mu \mathrm{PL})$ reveals a decrease in thermal quenching of the QDs integrated intensity compared to that of the WL. This difference in behaviour is due to the 3-D localization of carriers within the QDs preventing them from thermalization to nearby traps causing an increase in the internal quantum efficiency of the device. Excitation power dependent $\mu \mathrm{PL}$ shows a slower increase of the QDs PL signal compared to the WL PL which is believed to be due to the QDs saturation. (C) 2012 American Institute of Physics. [http://dx.doi.org/10.1063/1.4751434]
\end{abstract}

\section{INTRODUCTION}

Since the development of the GaN based blue light emitting diode (LED) by Nakamura, ${ }^{1}$ there have been constant efforts to utilize it for solid state light. However, since most of the commercial blue GaN LEDs are grown on sapphire pseudo-substrate $\left(\mathrm{Al}_{2} \mathrm{O}_{3}\right)$, the large lattice mismatch causes a rise in the defect density of up to $10^{9} \mathrm{~cm}^{-3}$ causing a severe decrease in the internal quantum efficiency (IQE) of the material. Furthermore, when high indium concentrations are incorporated in order to shift the emission wavelength towards red, for the generation of white light, the formation of micron-sized indium clusters causes a further decrease in the IQE of the LEDs. ${ }^{2}$ The incorporation of quantum dots (QDs) in indium-rich InGaN red LEDs is expected to increase the IQE through localizing the carriers away from the aforementioned nonradiative traps. ${ }^{3}$

In this paper, we report on the characterization of indium rich, red emission wavelength, self-organized InGaN/ GaN QDs compared to higher dimensional quantum structures through temperature and excitation power dependent micro-photoluminescence ( $\mu \mathrm{PL})$. In order to conduct a fair comparison, instead of comparing the 0-D QD structure to a 2-D quantum well (QW), QDs are compared with the underlying 2-D wetting layer (WL) and thus, dependence on the crystal parameters (e.g., defect concentrations) is not affecting the comparison. We observe how the QDs integrated intensity only suffers from a reduced thermal quenching due to a decrease in carrier thermalization. X-ray diffraction (XRD) measurements are performed to estimate the average indium concentration of the QDs and the WL.

\section{EXPERIMENT}

The sample used in the current study consists of a $500 \mu \mathrm{m}$ sapphire substrate with $1 \mu \mathrm{m}$ molybdenum deposited on the backside. The growth temperature is measured by an infrared pyrometer, calibrated by the reflection high energy electron diffraction (RHEED) transition $(7 \times 7$ to $1 \times 1)$ of $\mathrm{Si}(111)$ substrates. A $5 \mu \mathrm{m}$ silicon doped $\mathrm{GaN}$ is deposited at $740{ }^{\circ} \mathrm{C}$ at a rate of $180 \mathrm{~nm} / \mathrm{h}$ using hybrid vapor phase epitaxy (HVPE) in order to relieve the internal strain due to lattice mismatch at the sapphire-GaN interface. A $100 \mathrm{~nm}$ GaN layer is then grown using plasma assisted molecular beam epitaxy (MBE) where the RHEED pattern remained bright and streaky. The substrate temperature is then lowered to $520^{\circ} \mathrm{C}$ and the InGaN QDs growth is carried at a rate of $0.025 \mathrm{~nm} / \mathrm{s}$. The QDs are buried under $100 \mathrm{~nm}$ of GaN. Surface InGaN QDs are then grown and capped by $10 \mathrm{~nm} \mathrm{GaN}$ under the same conditions (refer to Fig. 1, inset a). ${ }^{4}$ The RHEED pattern shows a transition from streaky to spotty pattern during the QDs growth. The growth direction is along the [0001] polar c-axis causing the QDs as well as the WL to suffer from the quantum confined Stark effect (QCSE). ${ }^{5}$ The QDs are grown using the Stranski-Krastanov (SK) growth mode where a 2-D strained WL is first formed followed by 0-D QDs.

Symmetric XRD measurements $(\Omega-2 \theta$ scan of the 0002 plane) are performed to calculate the lateral lattice constant of the strained structures $\left(c_{I n G a N}\right)$. The thin WL and the QDs are assumed to be pseudomorphically grown $\left(a_{I n G a N}=a_{G a N}\right)$ and thus the indium content within the structures can be estimated by solving Hooke's law, ${ }^{6,7}$

$$
\left[c_{I n G a N}-c_{0}\right]+2 \frac{C_{13}(x) c_{0}(x)}{C_{33}(x) a_{0}(x)}\left[a_{I n G a N}-a_{0}\right]=0,
$$

\footnotetext{
${ }^{\text {a)} E l e c t r o n i c ~ m a i l: ~ b o o n . o o i @ k a u s t . e d u . s a . ~}$
} 


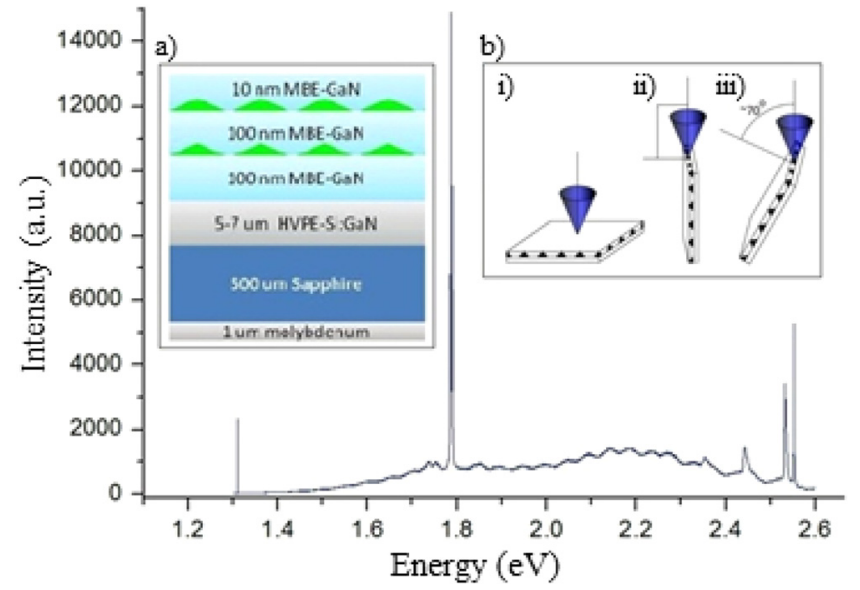

FIG. 1. $\mu \mathrm{PL}$ of the InGaN/GaN QD with a $473 \mathrm{~nm}$ laser at an excitation power density of $8400 \mathrm{~kW} / \mathrm{cm}^{2}$ at $78 \mathrm{~K}$. Inset a shows the sample schematics. Inset $\mathrm{b}$ shows the setup for $\mu \mathrm{PL}$ measurements from (i) the top, (ii) the side, and (iii) at an angle $70^{\circ}$ tilted off the vertical plane.

where $c_{0}$ and $a_{0}$ are the relaxed lattice constants and $C_{13}$ and $C_{33}$ are the stiffness coefficients of the InGaN structures. The downside of this technique is the underestimation of the indium content due to the crystal's partial relaxation. Two distinct peaks are easily resolved in the XRD spectrum, each corresponding to different In concentrations. Since QDs, grown under the SK growth mode, are formed to release internal strain energy, it is assumed that self-organized QDs have weaker internal strain fields than the WL. ${ }^{8}$ This difference causes the In content within the WL to start drifting towards the QDs under the effect of a strain induced migration. ${ }^{9}$ Thus, the low In concentration peak is attributed to the WL and the high In concentration peak is attributed to the QDs. The mole fraction of indium is estimated to be $27 \%$ for the QDs and $22.3 \%$ for the WL. ${ }^{7}$ These values are potentially underestimated since pseudomorphic strain conditions are assumed. ${ }^{6}$ A third XRD peak also appears which reflects the presence of pure InN clusters which is the reason for the degradation of the IQE of In rich InGaN LEDs. ${ }^{2}$ Excitation power dependent and temperature dependent $\mu \mathrm{PL}$ measurements are carried out using $473 \mathrm{~nm}\left(E_{\text {excitation }}=2.62 \mathrm{eV}\right)$ excitation laser. The average spot size of the laser is $7.07 \mu \mathrm{m}^{2}$. The temperature dependent $\mu \mathrm{PL}$ is measured at an excitation power density of $84 \mathrm{~kW} / \mathrm{cm}^{2}$ where the sample is cooled down to $78 \mathrm{~K}$ by an open loop cryostat using liquid nitrogen. The power dependent $\mu \mathrm{PL}$ is measured at room temperature.

\section{RESULTS AND DISCUSSION}

$\mu \mathrm{PL}$ at $78 \mathrm{~K}$ with a $84 \mathrm{~kW} / \mathrm{cm}^{2}$ excitation power is measured from the top plane (parallel to the c-axis as in inset b(i) of Fig. 1) and the results are presented in Fig. 1. The spectrum contains a broad signal and several sharp emission peaks. In order to know the origin of these sharp peaks, $\mu \mathrm{PL}$ is measured from the side plane with the laser being focused on the sapphire away from GaN (inset b(ii) of Fig. 1). The measured signal consists only of the sharp peaks in Fig. 1, which are attributed to chromium impurities present within the sapphire substrate. ${ }^{10}$ The oscillations present in the broad

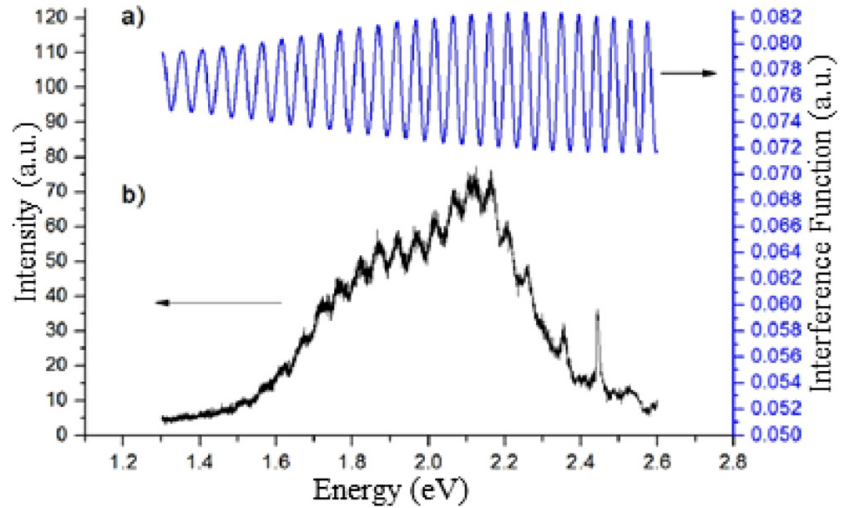

FIG. 2. (a) The interference function and (b) $\mu \mathrm{PL}$ of the InGaN/GaN QDs.

signal are due to Fabry-Perot resonances. Since the refractive indexes of air, Si:GaN, and sapphire are 1, 2.39, and 1.77, respectively, light generated in GaN oscillates between the GaN/air and GaN/Sapphire interfaces causing the interference fringes in Fig. 2(b). ${ }^{11}$ In order to remove these oscillations for reliable data analysis, we construct an interference function (Fig. 2(a)) based on the material optical properties. $^{12,13}$ The interference free PL is constructed by dividing the measured PL by the interference function and the results are presented in Fig. 3.

The sample $\mu \mathrm{PL}$ is best fitted with two broad distribution functions, one broad Gaussian distribution centered at $1.85 \mathrm{eV}$ (red domain) and a narrower Lorentzian distribution centered at $2.14 \mathrm{eV}$, and a series of sharp Lorentzian peaks with much lower intensity (Fig. 3). The low energy Gaussian distribution is attributed to the QDs, while the higher energy Lorentzian distribution is attributed to the WL for two main reasons. The higher In composition of QDs causes them to emit at lower energies than the WL. Also, the broad Gaussian curve reflects a large degree of inhomogeneity as compared to the narrower Lorentzian curve. The sharp high

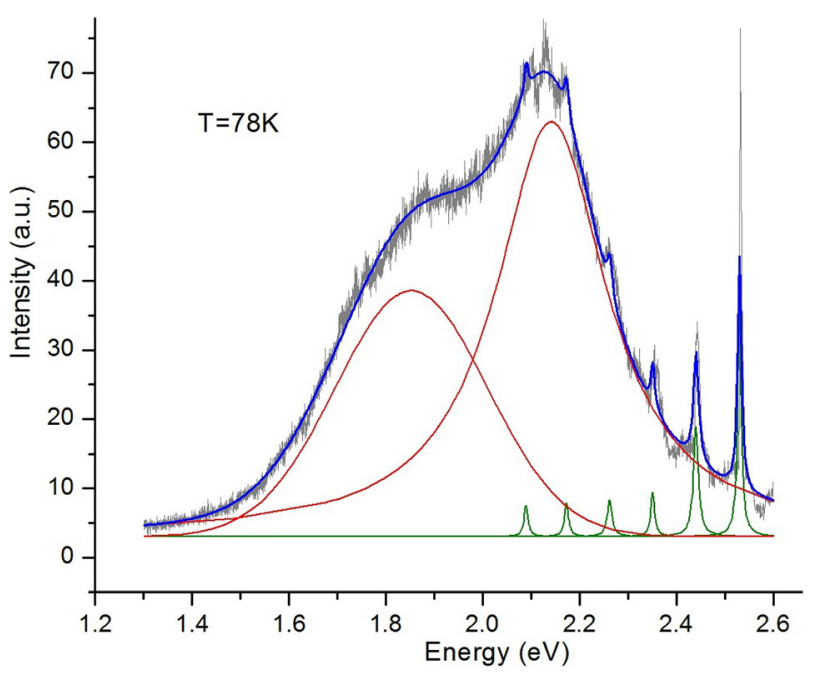

FIG. 3. $\mu \mathrm{PL}$ emission from the QD after subtracting the emission spectrum of the Sapphire substrate at $78 \mathrm{~K}$ and removing the Fabry-Perot oscillations (grey). The fitting curve (blue) consists of two broad high intensity distributions (red) attributed to the self-organized QDs and the WL and narrower lower intensity signals attributed to the phonon replicas of the excitation laser (green). 
energy peaks in Fig. 3 are attributed to the phonon replicas of the excitation laser $(2.62 \mathrm{eV})$ that are separated by around $91 \mathrm{meV}$, which is the characteristic energy of the longitudinal optical (LO) phonon in GaN. ${ }^{14}$

The emission energy is calculated by

$$
E_{0}=E_{g}+\Delta E-E_{p o l}-E_{e x}
$$

where $E_{g}$ is the InGaN bandgap energy, $\Delta E$ is the energy increase due to quantum confinement, $-E_{p o l}$ is the QCSE induced energy decrease due to internal polarization fields, and finally $E_{e x}$ is the exciton binding energy. ${ }^{15}$ For $E_{g}$, we use Vegard's law with $1.38 \mathrm{eV}$ and $1.482 \mathrm{eV}$ as the bowing parameters which are interpolated from published results for indium compositions of $27 \%$ and $22.3 \%$, respectively. ${ }^{16}$ The corresponding bandgap energies are calculated to be $2.4 \mathrm{eV}$ for the QDs and $2.56 \mathrm{eV}$ for the WL. Since the In mole fraction is underestimated, these values are expected to be blue shifted from the actual bandgap energies. For the QD confinement energy $(\Delta E)$, we use

$$
\Delta E=\frac{\pi^{2} \hbar}{2}\left(\frac{1}{m_{e}^{*}}+\frac{1}{m_{h}^{*}}\right) \times\left(\frac{1}{h^{2}}+\frac{2}{d^{2}}\right),
$$

where $\hbar$ is planks constant, $m_{e}^{*}$ and $m_{h}^{*}$ are the electron and hole effective masses, which are interpolated from the respective bulk material, respectively. Finally, $h$ and $d$ are the average QD height and width, respectively, which are estimated from high resolution transmission electron microscopy (HRTEM) micrographs. For calculating the WL confinement energy, the $2 / d^{2}$ term is dropped out since there is no lateral confinement. It is calculated that $\Delta E \mathrm{~s}$ are $86 \mathrm{meV}$ and $141 \mathrm{meV}$ for the QDs and the WL, respectively. Since a decrease in In composition translates into a shallower quantum traps, the calculated confinement energies are expected to be less than the actual confinement energies. $E_{p o l}$ is approximated to be equal to $h . F$, where $F$ is the built in electric field given by

$$
F=\frac{P_{P Z}^{I n G a N}+P_{S P}^{I n G a N}-P_{S P}^{G a N}}{\epsilon_{I n G a N} \epsilon_{0}} .
$$

$P_{P Z}$ and $P_{S P}$ are the built in piezoelectric and spontaneous polarizations, respectively. The spontaneous polarization of the InGaN ternary alloy is interpolated from the spontaneous polarizations of $\mathrm{GaN}$ and $\mathrm{InN}\left(P_{S P}^{G a N}=0.029 \mathrm{Q} / \mathrm{m}^{2}\right.$, $\left.P_{S P}^{I n G a N}\right|_{O D}=-0.018 Q / \mathrm{m}^{2}$, and $\left.\left.P_{S P}^{I n G a N}\right|_{W L}=-0.025 Q / \mathrm{m}^{2}\right) .{ }^{17}$ The piezoelectric polarization is calculated from the internal strain values by

$$
\begin{aligned}
& \left.P_{P Z}^{I n G a N}\right|_{x}=e_{31}\left(\epsilon_{x x}+\epsilon_{y y}\right)+e_{33} \epsilon_{z z}, \\
& \left.P_{P Z}^{I n G a N}\right|_{y}=e_{51} \epsilon_{z x}, \\
& \left.P_{P Z}^{I n G a N}\right|_{z}=e_{51} \epsilon_{y x},
\end{aligned}
$$

where $\epsilon_{i j}$ and $e_{i j}$ are the internal strain values and the piezoelectric constants, respectively. ${ }^{18}$ Assuming a biaxial strain field, the piezoelectric polarization fields can be approximated to

$$
\begin{aligned}
& \left.P_{P Z}^{I n G a N}\right|_{x}=\left(2 e_{31}-2 e_{33} \frac{C_{13}}{C_{33}}\right) \times \frac{a_{G a N}-a_{I n G a N}}{a_{I n G a N}} \\
& \left.P_{P Z}^{I n G a N}\right|_{y}=\left.P_{P Z}^{I n G a N}\right|_{z}=0,
\end{aligned}
$$

where $C_{i j}$ are the elastic constants which are linearly interpolated from bulk materials and $a_{G a N}$ and $a_{\text {InGaN }}$ are the basal lattice constants of $\mathrm{GaN}$ and $\mathrm{InGaN}$, respectively. ${ }^{19}$ The calculated values for $E_{p o l}$ are $0.622 \mathrm{eV}$ and $0.539 \mathrm{eV}$ for the QDs and the WL, respectively. The magnitude of the actual values should be lower than calculated due to the partial relaxation in the crystal which lowers the built in piezoelectric field and thus, decreasing the QCSE. As for $E_{e x}$, it is neglected since the large inhomogeneously broadened full width at half maximum (FWHM) is around $397 \mathrm{meV}$ (Fig. 3), which is more than 10 times the expected exciton binding energy in GaN. ${ }^{20}$ Summing up all the previous energy shifts, we get $\left.E_{0}\right|_{O D}=1.86 \mathrm{eV}$ and $\left.E_{0}\right|_{W L}=2.16 \mathrm{eV}$, which are blue shifted by approximately $10 \mathrm{meV}$ for the QDs and $20 \mathrm{meV}$ for the WL from the measured values. This discrepancy is expected to be due to a higher In composition than estimated from the XRD analysis.

Instead of the pseudomorphic strain assumption, crystal relaxation $\left(a_{I n G a N}>a_{G a N}\right)$ is included in the XRD analysis and energy calculations. The crystal relaxation is let to increase till the calculated total energy coincides with the measured emission energy. The In mole fractions are then recalculated and the results among with the calculated energies are tabulated in Table I, columns (b). The recalculated values which represent more realistic estimates show indeed an increase in the In compositions. Two main points are to be noted. First, the underestimated In compositions (Table I, columns (a)) blue shifts $E_{g}$ which causes an overall increase in the calculated emission energy. Also, since pseudomorphically strained structures have stronger internal polarization fields than partially relaxed structures, the total calculated energy gets red shifted from its actual value due to the amplified QCSE. By comparing the errors in $E_{g}$ and $E_{\text {pol }}$, it becomes clear that the blue shifts outweigh the red shifts explaining why the total calculated energy is blue shifted from the measured value.

TABLE I. (a) Indium compositions calculated from the XRD data, based on the pseudomorphic assumption, and the associated energy values are tabulated. (b) By varying the crystal relaxation, the total emission energy is forced to coincide with the measured emission energy. The corresponding In compositions and the recalculated energy constituents are tabulated. The difference between the two calculations is presented in the error columns.

\begin{tabular}{lcccccc}
\hline \hline & \multicolumn{3}{c}{ QD } & & \multicolumn{2}{c}{ WL } \\
\cline { 2 - 3 } & (a) & (b) & Error & (a) & (b) & Error \\
\hline In comp. (\%) & 27 & 27.7 & & 22.3 & 23.3 & \\
$E_{g}(\mathrm{eV})$ & 2.4 & 2.37 & 0.03 & 2.56 & 2.52 & 0.04 \\
$\Delta E(\mathrm{meV})$ & 86.3 & 86.6 & -0.3 & 141 & 142 & -1 \\
$\left|E_{\text {pol }}\right|(\mathrm{meV})$ & 622 & 610 & 12 & 539 & 525 & 14 \\
Total energy $(\mathrm{eV})$ & 1.86 & 1.85 & 0.01 & 2.16 & 2.14 & 0.02 \\
\hline \hline
\end{tabular}


To gain deeper understanding of the carrier dynamics within the QDs sample, we perform $\mu \mathrm{PL}$ measurements while varying the temperature from $78 \mathrm{~K}$ to $313 \mathrm{~K}$. The excitation power is kept as low as possible in order to prevent QD saturation. At $78 \mathrm{~K}$, the WL emission is higher than the QDs, which is due to the higher density of states present in the WL as compared to the QDs. However, as the temperature starts to rise, the WL $\mu \mathrm{PL}$ decreases much faster than the QDs PL, till at $313 \mathrm{~K}$, the QDs emission has a higher intensity than the WL (Fig. 4). The $\mu$ PL curves are fitted with the distributions presented in Fig. 3 and the integrated intensity of the Gaussian and Lorentzian distributions are calculated at different temperatures and the results are plotted in Fig. 5. To present an explanation to the fast decrease in the WL emission compared to the QDs emission, we use the thermal activation model (inset of Fig. 5). ${ }^{21}$

The model presents the case when an electron is at an excited state $|e\rangle$ (either in the QDs or in the WL), then relaxes down to the ground state $|g\rangle$. It either radiatively relaxes down to the valence band $\mid v>$ contributing to the IQE of the structure or it gets thermalized by a phonon to a nearby trap $\mid t>$ separated by energy $\mathrm{E}$ where the carrier undergoes nonradiative recombination decreasing the IQE. ${ }^{22}$ Since phonon density increases with increasing temperature, the thermalization process is expected to increase with temperature. The temperature dependent PL integrated intensity, $I(T)$, is given by

$$
I(T)=n R_{\text {spont }}=\frac{I(0)}{1+a e^{-E / K_{B} T}}
$$

where $n$ and $R_{\text {spont }}$ are the QD carrier concentration and the spontaneous recombination rate, respectively. $I(0)$ is the relaxation rate at $0 \mathrm{~K}$ and $K_{B}$ is the Boltzmann constant. Solid lines in Fig. 5 show the expectation results from the thermal activation model which is in a good agreement with the measured results implying that what causes a decrease in the PL signal is indeed carriers thermalization to nearby

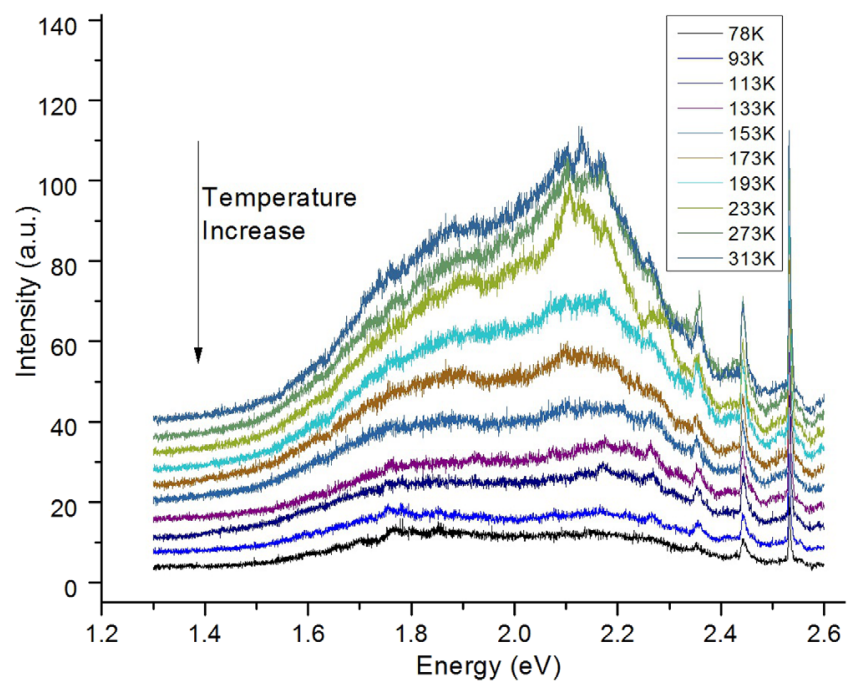

FIG. 4. Stacked $\mu$ PL curves of the InGaN/GaN QD at different temperatures starting from $78 \mathrm{~K}$ till $313 \mathrm{~K}$. The sample is excited by a $473 \mathrm{~nm}$ laser with a power density of $84 \mathrm{~kW} / \mathrm{cm}^{2}$.

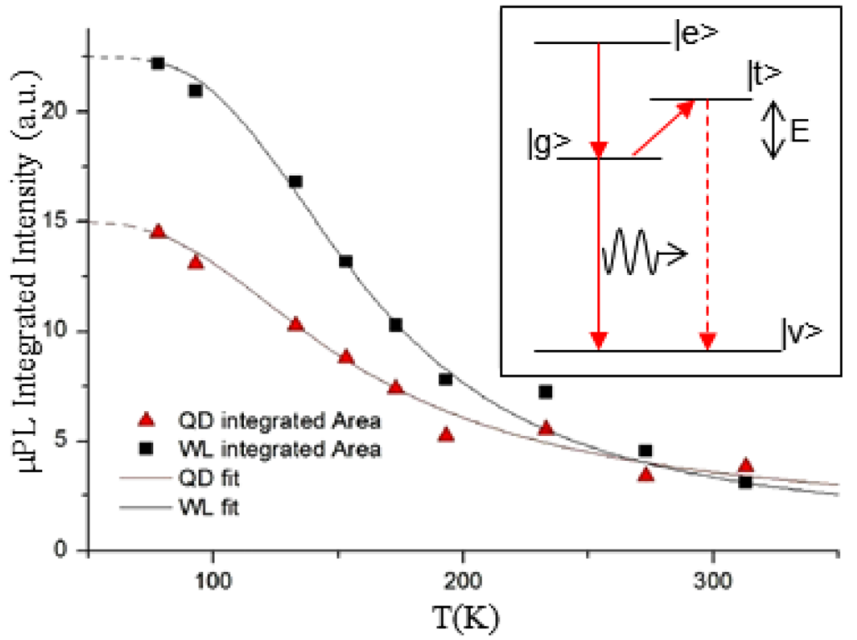

FIG. 5. Solid triangles and squares represent the measured integrated intensity for the PL emission from the QDs and the WL, respectively. The solid lines represent the excepted temperature dependence of the thermal activation model. The inset is a presentation of the thermal activation model.

traps. Since the QDs $\mu$ PL signal does not fall as fast as the WL $\mu \mathrm{PL}$ signal, this means that once carriers relax down into a QD, they do not get as easily thermalized to traps as when they relax to the WL. This is in agreement with the effect of the three dimensional localization of QDs which prevents carriers from being trapped by defects. ${ }^{3}$ This is not the case for the WL, where carriers can diffuse laterally increasing the probability of encountering a defect where they undergo nonradiative recombinations. Thus, QDs based InGaN LEDs are expected to have higher IQE than QWs based InGaN LEDs.

Power dependent $\mu \mathrm{PL}$ measurements for the QDs with their underlying WL are presented in Fig. 6 where the maximum excitation power is $84 \mathrm{~kW} / \mathrm{cm}^{2}$. The measured spectrum is interference free since it was collected from the Brewster angle (inset b(iii)) of Fig. 1) with a polariser to filter out the S-polarization of the $\mu \mathrm{PL}$ signal. ${ }^{13}$ The observed

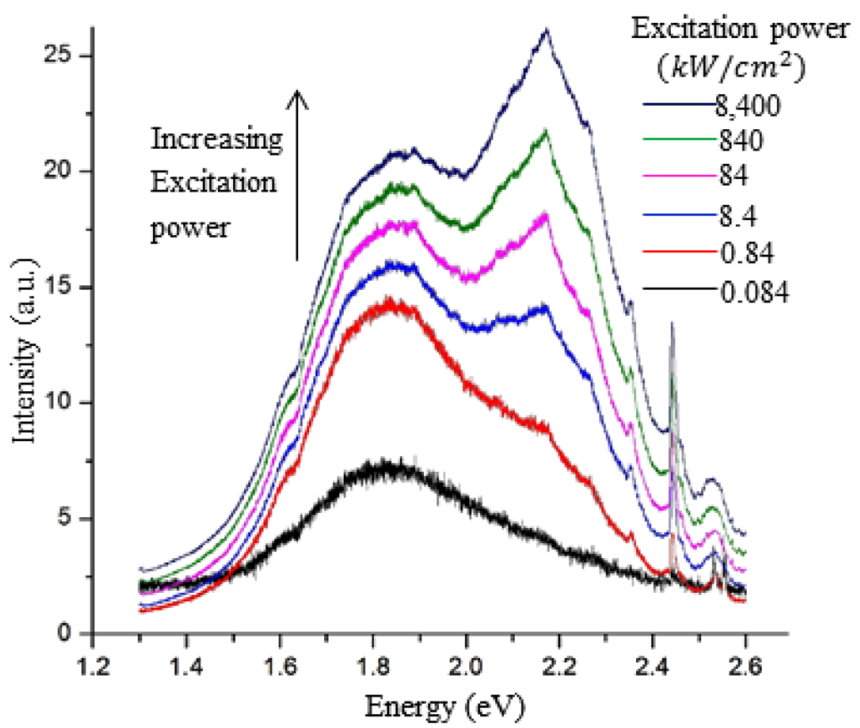

FIG. 6. Power dependent $\mu \mathrm{PL}$ at room temperature. Maximum excitation power is $8400 \mathrm{~kW} / \mathrm{cm}^{2}$ and lowest is $0.084 \mathrm{~kW} / \mathrm{cm}^{2}$. 
kinks are due to the phonon replicas of the excitation laser. As the excitation power increases, the WL emission increases at a higher rate than the QDs emission. Since all the curves in Fig. 6 are collected at the same temperature, the thermal activation model does not explain these differences. One plausible explanation is that as the electric field of the photo-generated carriers screens the internal polarization electric field, the QCSE decreases. This allows for a higher overlap between the electron and hole wave-functions which increases the emission intensity. ${ }^{23}$ Since the QDs internal stress is more isometric than the WL, the WL is suffering from a stronger QCSE and thus, as the excitation power increases, the $\mathrm{WL} \mu \mathrm{PL}$ recovers from the QCSE causing its intensity to increase. ${ }^{24,25}$ However, since no blue shift is observed with increasing excitation power, this cannot be the main mechanism behind the faster increase of the WL intensity.

As the excitation power increases, the WL can keep accommodating the extra photo-generated carriers while the QDs get saturated due to their lower density of states compared to the WL. ${ }^{15}$ Thus, the WL $\mu \mathrm{PL}$ intensity keeps increasing over the QDs $\mu \mathrm{PL}$ intensity. The later reason seems to be the main mechanism behind the slower increase of the QD intensity since it does not cause any change in the emission energy. In order to overcome the saturation problem, a higher density of QDs can be grown which will further increase the IQE of the sample allowing the production of high efficiency long wavelength indium rich InGaN/GaN LEDs.

\section{CONCLUSION}

Indium rich, red shifted, self-organized InGaN/GaN QDs offering 3-D carrier localizations are characterized against the WL with QW like characteristics. Optical studies reveal the ability of QDs to confine carriers preventing them from getting thermalized into nonradiative traps caused by indium clusters. Temperature dependent $\mu \mathrm{PL}$ reveals a slow thermal quenching in the QDs $\mu \mathrm{PL}$ intensity. Self assembled QD nano-structure allows the production of high efficiency long wavelength InGaN/GaN red LED that can be incorporated with the shorter wavelength InGaN/GaN green and blue LED paving the way for the production of white light LED.

\section{ACKNOWLEDGMENTS}

The authors are grateful for the generous funding from the KAUST fellowship program. This work was also supported by a joint program between KAUST and University of Michigan, Ann Arbor, under KAUST Academic Excellence Alliance (AEA) 2010 Grant.

${ }^{1}$ S. Nakamura, M. Senoh, N. Iwasa, and S.-I. Nagahama, Jpn. J. Appl. Phys. 34, L797 (1995).

${ }^{2}$ Y. H. Cho, S. K. Lee, H. S. Kwack, J. Y. Kim, K. S. Lim, H. M. Kim, T. W. Kang, S. N. Lee, M. S. Seon, O. H. Nam, and Y. J. Park, Appl. Phys. Lett. 83, 2578 (2003).

${ }^{3}$ S. C. Davies, D. J. Mowbray, F. Ranalli, P. J. Parbrook, Q. Wang, T. Wang, B. S. Yea, B. J. Sherliker, M. P. Halsall, R. J. Kashtiban, and U. Bangert, Appl. Phys. Lett. 95, 111903 (2009).

${ }^{4}$ M. Zhang, P. Bhattacharya, and W. Guo, Appl. Phys. Lett. 97, 011103 (2010).

${ }^{5}$ D. Williams, S. Schulz, A. D. Andreev, and E. O'Reilly, IEEE J. Sel. Top. Quantum Electron. 15, 1092 (2009).

${ }^{6}$ K. O’Donnell, J. Mosselmans, R. Martin, S. Pereira, and M. White, J. Phys.: Condens. Matter 13, 6977 (2001).

${ }^{7}$ S. Pereira, M. R. Correia, T. Monteiro, E. Pereira, E. Alves, A. D. Sequeira, and N. Franco, Appl. Phys. Lett. 78, 2137 (2001).

${ }^{8}$ B. Damilano, N. Grandjean, S. Dalmasso, and J. Massies, Appl. Phys. Lett. 75, 3751 (1999).

${ }^{9}$ Q. Xie, A. Madhukar, P. Chen, and N. P. Kobayashi, Phys. Rev. Lett. 75, 2542 (1995).

${ }^{10}$ M. Toth and M. R. Phillips, Appl. Phys. Lett. 75, 3983 (1999).

${ }^{11}$ C. Hums, T. Finger, T. Hempel, J. Christen, A. Dadgar, A. Homann, and A. Krost, J. Appl. Phys. 101, 033113 (2007).

${ }^{12}$ R. T. Holm, S. W. McKnight, E. D. Palik, and W. Lukosz, Appl. Opt. 21, 2512 (1982).

${ }^{13}$ E. Namvar and M. Fattahi, J. Lumin. 128, 155 (2008).

${ }^{14}$ X. B. Zhang, T. Taliercio, S. Kolliakos, and P. Lefebvre, J. Phys.: Condens. Matter 13, 7053 (2001).

${ }^{15}$ J. Bai, Q. Wang, T. Wang, A. G. Cullis, and P. J. Parbrook, J. Appl. Phys. 105, 053505 (2009).

${ }^{16}$ P. G. Moses, M. Miao, Q. Yan, and C. G. Van de Walle, J. Chem. Phys. 134, 084703 (2011).

${ }^{17}$ F. Bernardini and V. Fiorentini, Phys. Rev. B 64, 085207 (2001).

${ }^{18}$ Y. R. Wu, Y. Y. Lin, H. H. Huang, and J. Singh, J. Appl. Phys. 105, 013117 (2009).

${ }^{19}$ F. Yam and Z. Hassan, Superlattices Microstruct. 43, 1 (2008).

${ }^{20}$ J. Shi, Solid State Commun. 124, 341 (2002).

${ }^{21}$ M. Sugisaki, H. W. Ren, S. V. Nair, J. S. Lee, S. Sugou, T. Okuno, and Y. Masumoto, J. Lumin. 87-89, 40 (2000).

${ }^{22}$ Y. Masumoto and T. Takagahara, Semiconductor Quantum Dots: Physics, Spectroscopy and Applications (Springer, 2010).

${ }^{23}$ V. Ranjan, G. Allan, C. Priester, and C. Delerue, Phys. Rev. B 68, 115305 (2003).

${ }^{24}$ Y. Narukawa, Y. Kawakami, S. Fujita, and S. Nakamura, Phys. Rev. B 59, 10283 (1999).

${ }^{25}$ J. H. Davies, J. Appl. Phys. 84, 1358 (1998). 